\title{
Big Bang not yet dead but in decline
}

The latest measurements of the Hubble constant make the Big Bang account of the origin of the Universe more dependent on the coincidence of numbers than it has so far been. But it remains the only theory in the field.

Is there a crisis in cosmology, or is it that the latest measurement of the Hubble constant is yet another of those numerical disagreements that plague the field from time to time? That is the question inevitably prompted by last week's article by N.R. Tanvir et al.(Nature 377, 27-31; 1995). Their chief conclusion, based on the accurately known recession speed of the Coma cluster of galaxies, is that the Hubble constant (otherwise $H_{0}$ ) implies an age of the Universe much smaller than the known ages of the stars in globular clusters in our Galaxy.

The obvious question is how that can be if the stars in the Galaxy were formed within the lifetime of the Universe. The obvious answer is that the result, the third of its kind in under a year, makes a nonsense of the standard Big Bang view of how the Universe began. But even those who are persuaded on other grounds that the Big Bang is a fairy story, perhaps for no better reason than that it is "too good to be true", had better conduct themselves with circumspection in the months ahead.

When the Hubble Space Telescope (HST) was built in the 1970s, it was generally understood that one of its chief purposes was to help construct a better distance-scale in the Universe. At that stage, it was also widely understood that the identification of Cepheid variables in more distant galaxies would play a crucial part in the process. (Cepheid variables are late-type stars whose period of oscillation is unambiguously related to their absolute luminosity.) Two of the three potentially disconcerting articles published in the past year have indeed been based on data gathered by the HST. All of them have turned on data gathered from Cepheids.

The three values of $H_{0}$ published in the past year are interesting in themselves. M.J. Pierce et al., using the Keck telescope on Hawaii, reached the conclusion that $H_{0}$ is $87 \pm 7 \mathrm{~km} \mathrm{~s}^{-1} \mathrm{Mpc}^{-1}$ (where $1 \mathrm{Mpc}$ is a million paresecs or roughly three times as many light-years). That result hung on measurement of Cepheid variables in the galaxy NGC4571, one of the galaxies of the Virgo cluster (Nature 371, 385-389; 1994). A few weeks later, there appeared Wendy L. Freedman et al. (Nature 371, $757-761 ; 1994)$ with a value of $H_{0}=80 \pm$ $17 \mathrm{~km} \mathrm{~s}^{-1} \mathrm{Mpc}^{-1}$, based on HST mea- surements of Cepheids in another galaxy in the Virgo cluster, called M100. Now we have $69 \pm 8 \mathrm{~km} \mathrm{~s}^{-1} \mathrm{Mpc}^{-1}$.

The temptation to regard either the simple average $\left(78.5 \mathrm{~km} \mathrm{~s}^{-1} \mathrm{Mpc}^{-1}\right)$ or some weighted average of these values as a better approximation to the "true" value should be resisted. All three groups of authors are careful to emphasize that their results entail chains of inference that may be false. The Virgo measurements suppose that the two galaxies concerned are near the core of the cluster, for which there is circumstantial evidence but no proof. Tanvir et al. rely on a different chain of inference: Cepheids in M96, a member of the Leo I galaxy cluster at a distance of about $12 \mathrm{Mpc}$, and the use of "consensus" estimates of relative distance to get from there to the Coma cluster, which is compact enough and far enough away $(105 \pm 12 \mathrm{Mpc})$ for the motion of individual galaxies to be irrelevant to the recession of the cluster as a whole. At this stage, it must be very hard to be sure that there is now some common false assumption in all three chains of inference.

How to resolve the difficulty? More data are the obvious solution. With HST in such good shape, there is no reason why it should not be used to look for Cepheids in almost all the galaxies of the Virgo cluster, for example, getting rid in the process of the continuing uncertainty about the structure of the Virgo cluster, which would be worthwhile in itself.

Those with their knife into the Big Bang should also reflect that if the value of $H_{0}$ eventually settles down near the values recently published, it will be necessary to come to an understanding of why so many other measurements have given values that are numerically roughly half as big. Those responsible are not, of course, slouches. Among others, they include Alan T. Sandage, who has for decades been poring over Palomar Mountain plates with the objective of defining a distance scale for the Universe.

It is also crucial that the apparent conflict between the recent measurements of $H_{0}$ and the age of the Universe depends on assumptions about the density of the Universe as well as on the particular solution of Einstein's equations used to describe the evolution of the Universe. The lower the density of matter in the
Universe, the more easily it is possible to reconcile a large value of $H_{0}$ with the existence of old stars. Freedman et al. put the point clearly last year; their value of $H_{0}$ is compatible with stars as old as 16 billion years only if the density of the Universe is a few per cent of the socalled "critical density" separating indefinite expansion from expansion followed by collapse.

That is what the density of the Universe seems in reality to be if its mass is represented by the visible galaxies and if there is no substantial amount of "missing mass". Many of those who are inherently suspicious of the Big Bang have been persuaded into that frame of mind by realizing that the search for the missing mass has much in common with the fruitless search for the lumeniferous aether at the turn of the present century; some of them may now suspend their disbelief.

That does not eliminate the need for some hidden source of mass to account for the way in which the outer parts of spiral galaxies (such as ours) rotate more quickly than can be explained by the visible mass concentrated in their inner regions. There is also the problem of what holds clusters of galaxies together gravitationally; the simplest assumption is that there is invisible mass in the shape of intergalactic gas (which is sometimes made visible by its X-ray emission when made very hot by collapse or by shock waves). Nobody may know what the missing mass consists of, but there can be no doubt of the reality of some of it.

In short, the new measurements of $H_{0}$ are not the death of the Big Bang, but merely a further sign of its fragility. There is also ample evidence on the other side, of course - the cosmic abundance of isotopes such as deuterium and ${ }^{3} \mathrm{He}$ for example. Naturally, it is not comforting that the truth on such a crucial question as the origin of the Universe should hang on numbers; qualitative arguments would be preferable. Perhaps the time has come for a reinvestigation of the origin of globular clusters, which contain the embarrassing oldest stars. The minute it is suggested that these structures are so exceptional that they must be survivors from an earlier stage in the evolution, the Big Bang will have given way to continuous creation. That will be a turn-up for the book.

John Maddox 